\section{$\$$ Research Square}

\title{
Prognostic nomograms for predicting overall survival of Paget 's disease
}

\section{Wei Li}

The second hospital,University of South China https://orcid.org/0000-0002-8503-4634

Kun Fang ( $\square$ k99ft|@163.com )

https://orcid.org/0000-0003-3922-4434

\section{Guo Huang}

The second hospital,University of South China

\section{You-quan Wang}

The second hospital,University of South China

\section{Hong Cao}

The second hospital,University of South China

\section{Lin Long}

The sceond hospital,University of South China

\section{Dan Li}

The second hospital,University of South China

\section{Tian Zhu}

The second hospital,University of South China

\section{Kang-yun Tang}

The second hospital,University of South China

\section{Li-zhi Su}

The second hospital,University of South China

\section{Primary research}

Keywords: Paget's Disease, SEER database, nomogram, Prognostic,Overrall Servival

Posted Date: March 10th, 2020

DOI: https://doi.org/10.21203/rs.3.rs-16545/v1

License: (9) This work is licensed under a Creative Commons Attribution 4.0 International License. Read Full License 


\section{Abstract}

Background The death rate of metastatic breast cancer in patients with mammary Paget's disease and underlying cancer is $61.3 \%$, with a 10 -year cumulative survival rate of $33 \%$. The aim of this investigation was to develop a reliable nomogram to predict Female Paget's Disease patient, thus helping clinical diagnosis and treatment.

Methods Date of patients diagnosed with Paget's Disease between 2004 to 2014 were downloaded from the Surveillance, Epidemiology, and End Results (SEER)database. Univariate and multivariate cox analysis were used to identify independent prognostic factors, which were used to construct nomograms to predict the probabilities of OS. The performance of the nomogram was assessed by C-indexes, receiver operating characteristic (ROC) curves and calibration curve. Decision curve analysis (DCA) was used to compare clinical usage between the nomogram and Adjusted AJCC staging system.

Results Based on the univariate and multivariate cox analysis, features that correlated with survival outcomes were used to establish nomograms for OS prediction. The nomograms showed favorable sensitivity at predicting 1-,3-, and 5-year OS, with a C-index of $0.795(95 \%$ confidence interval $(\mathrm{Cl}) 0.773-$ 0.818 ) for OS. Calibration curves and ROC curves revealed excellent predictive accuracy. Finally, the 3and 5-year DCA curves yielded larger net benefits than the Adjusted AJCC stage.

Conclusions In conclusion, we have successfully established an effective nomogram to predict OS in Paget's Disease patient, which can help clinicians in determining the appropriate therapy strategies for individual PD patients.

\section{Background}

Breast cancer is the most common malignancy in women worldwide [1]. Although the prognosis of breast cancer patients is generally favorable due to early detection and the comprehensive treatment, $20 \%-30 \%$ of patients would still develop distant metastases and cases with progressive stage only have a median two-year survival time [2-4]. Paget's disease (PD) of the breast is a relatively rare clinical manifestation with a reported incidence of $1 \%-3 \%$ of all primary breast cancers (BCs) [5-8]. It is often present in ductal carcinoma in situ (DCIS) and/or invasive ductal carcinoma (IDC) [9-11]. Clinically, patients with PD of the breast typically present with nipple or areolar skin changes with or without associated with palpable mass in the breast. Skin changes in the NAC may include itching, erythema, scaly or flaky skin, bloody nipple discharge, nipple erosion or ulceration, and nipple retraction [12]. Because the inchoate symptoms of PD are not standard and PD is commonly treated initially as a benign of dermatologic condition, the diagnosis is often delayed for months or longer [13].

Nomograms, as reliable and convenient prognostic tools, have been widely used to predict specific outcomes in clinical oncology. They can quantitatively predict the prognosis in certain patients with known and vital prognostic factors, and illustrate the numerical probability of clinical outcomes[14,15] The American Joint Committee for Cancer (AJCC) staging system is a tool commonly used by 
oncologists to predict disease progression and design therapeutic strategies [16,17]. However, given the various factors that influence the course of cancer, prognosis based on the AJCC staging alone is unreliable. This study aims to establish a comprehensive prognostic evaluation of PD by building a nomogram to understand the risk factors and prognosis better. We also propose to compare the prognostic value of the nomogram with that of the Adjusted AJCC staging system based on patient data available in the Surveillance, Epidemiology, and End Results (SEER) database.

\section{Methods}

\section{Patient eligibility and study variables}

The present study was performed at the Yinchuan Women and Children's Hospital and the Second Hospital of University of South China. Ethics requirement was not required because of no direct involvement with human participants or animals. Patients diagnosed with PD from 2004 to 2014 were enrolled in from SEER database. Patients were included if they fulfilled the diagnosis of breast cancer, and the histological type was confirmed as PD (8040/3-8443/3) based on International Classification of Diseases for Oncology (ICD-0-3). A retrospective cohort research was performed using data from SEER database. The SEER ${ }^{\star S}$ tat software (Version 8.3.6, National Cancer Institute, Washington DC, USA) was used to access the database and a data-use agreement was signed for this study. Patients were enrolled in this study according to the following inclusion and exclusion criteria. Inclusion criteria: (1) Patients were diagnosed with Paget's disease; (2) Breast cancer was the first primary malignancy;(3) Female patient. Exclusion criteria: (1) Metastatic status was unknown; (2) Follow-up data was missing; (3) Information about molecular subtype was unknown. Total 12 variables were selected in the present study, including the race, Laterality, Grade, AJCC 6th Stage (1988-2015) I-IV, Hormone receptor status, human epidermal growth factor receptor-2 (HER2) status, lymph node status, surgery, radiotherapy, chemotherapy, Marital status.

\section{Statistical analysis}

\section{Construction and validation of nomogram model}

Cox proportional hazard $(\mathrm{PH})$ regression model was used to calculate the hazard ratio (HR) along with the corresponding $95 \%$ confidence interval $(\mathrm{Cl})$ for each potential risk factor. Backward stepwise in Cox $\mathrm{PH}$ regression model resulted in the successful identification of all independent risk indicators. A nomogram model was constructed based on these independent risk factors. The nomogram was established for predicting 1-,3- and 5-year OS using the package of rms in R software version 3.6.1, which included all independent prognostic indicators. A two-sided $\mathrm{P}<0.05$ was considered statistically significant. In validation, the concordance index (C-index) was used for the measurement of nomogram between performance and predicted results. Calibration plots were constructed to validate the accuracy and reliability of the nomograms for OS by comparing the nomogram-predicted and actual survival rates determined in a Kaplan-Meier analysis with 1000 bootstrap samples [18]. Receiver operating 
characteristics (ROCs) curve was used for the sensitivity and specificity of nomogram. Furthermore, Decision curve analysis (DCA) was used to compare clinical usage between the nomogram and AJCC staging system.

\section{Results}

\section{Patient Characteristics}

We evaluated 1,440 female patients with Paget 's disease from 2004 to 2014. Among these populations, the majority of patients were over 50 -year-old (67.7\%), white (76.5\%), and married (51.9\%). In addition, the degree of differentiation of grade II and III tumors accounted for 28.3 and $60.8 \%$ of all cases, respectively. Among these patients, lymph node invasion-positive patients were (37.4\%). Among Paget's disease patients, the corresponding rate is ER- / PR- (46.9\%), ER + / PR- (17.9\%), ER- / PR + (2.7\%), ER + / PR + (33\%), and HER2 positive (19.4\%). In addition, the vast majority of patients with PD underwent surgery, and Table 1 showed the details.

\section{Screening For Pd Prognostic Factors}

Based on univariate and multivariate Cox proportional hazard regression analysis, we identified several independent prognostic factors. Age of Diagnosis [(51-70)( hazard ratio, HR = 1.95, $\mathrm{P}<0.001),(>70)$ (hazard ratio, $\mathrm{HR}=5.93, \mathrm{P}<0.001$ )];Race (None-white) (hazard ratio, $\mathrm{HR}=1.29, \mathrm{P}=0.044$ ), phase II AJCC $(H R=2.4, P=0.023)$, level III AJCC $(H R=6.33, P<0.001)$, level IV AJCC $(H R=16.93, P<0.001)$, radiotherapy $(H R=0.76, P=0.045)$ and receive surgery $(H R=0.47, P<0.005)$, chemotherapy $(H R=0.48, P$ $<0.001$ ) were related to OS in PD patients (Table 2) ).

\section{Nomograms Construction}

Based on the independent prognostic factors obtained from the Cox analysis, nomograms were constructed to predict OS at 1 year, 3 years, and 5 years (Fig. 1). The nomograms demonstrated that AJCC staging had the greatest impact on prognosis, followed by age, surgery, chemotherapy, marital status, radiation therapy, and race. Each level of each variable was assigned a score of a score level. By adding up the scores for each selected variable, we calculated the total score. The predictions corresponding to this total score then helped to estimate the 1-, 3-, and 5-year OS for each patient.

\section{Validation Of The Nomogram}

The calibration plots for the probability of 1-, 3- and 5-year OS demonstrated good concordance between the nomogram predictions and actual observations in the discovery set (Fig. 2) 


\section{Performance of the nomogram}

The C-indexes for OS were 0.795 . The AUC values for nomogram $(0.86,0.83$, and 0.82 for $1-, 3$-, and 5 year OS, respectively) and AJCC $(0.72,0.75$, and 0.69$)$ indicated the good discriminative ability of the nomogram, as shown in Fig. 3. These results indicate that our nomogram has a greater potential for accurately predicting prognosis compared to the AJCC stage model.

\section{Clinical Application}

DCA was performed to compare the clinical usability and benefits of the nomogram with that of the traditional AJCC stage. As shown in Fig. 4, compared to the AJCC stage model, the new nomogram's 3and 5-year DCA curves showed larger net benefits across a range of death risk in the validation cohort.

\section{Discussion}

Paget's disease (PD) of the breast is a relatively rare clinical manifestation with a reported incidence of $1-3 \%$ of all primary breast cancers (BCs) [5-8]. PD occurs mainly in women, but can also occur in men much more rarely $[19,20]$. Typical manifestations are pruritus, erythema, scaly skin, nipple hemorrhage, nipple erosion, or ulcers in the nipple and areola area [21], and occasionally there are cases of nipple loss. Delayed treatment often misdiagnosed as benign disease such as eczema affects prognosis [22-24]. There is no independent guideline for the treatment of PD, because it is often accompanied with diabetes, and the treatment plan is based on the associated Tumor formulation. The current research on the total sample size of PD complications replaces the clinical basis of the disease. There is not much data in this case. However, owing to the low incidence, there has been no patient-controlled study that conducted survival analysis of Paget's disease. Therefore, it is necessary to use Cox regression analysis and develop line charts to provide a comprehensive predictive model that includes not only systematic demographic information, but also surgical treatment and other clinical parameters to predict the risk of PD.

Although the AJCC staging system can help to predict the prognosis of PD patients [25], it ignores some important risk factors, such as age, ethnicity, and marital status. A nomogram is a convenient graphical representation of a mathematical model. It provides an intuitive way to combine important factors and predict a specific endpoint. Nomogram is also a reliable tool for quantifying risk and widely used in applied tumor prognosis. A well-developed clinical nomogram is a popular decision-making tool that can be used to predict the outcome of an individual and benefit both clinicians and patients [26]. Prognostic nomograms are the visualization of complicated statistical model that were used to predicting individual survival outcomes, and numerous advantages were observed in prognostic nomograms including good accuracy, user-friendliness and comprehensibility, allowing for wide application in clinical practice [2729]. In our study, we built a more comprehensive model based on a combination of various risk factors to better predict the prognosis of PD patients. Divided with the traditional AJCC staging system, based on 
the seven variables of age, race, AJCC stage, marital status, race, radiotherapy and surgery, and the traditional AJCC staging system ranking, it can better predict and assess the prognosis of PD patients.

Paget's disease is almost always associated with an underlying in situ or invasive cancer or both [30]. In previous study, increasing age and being unmarried (divorced, separated, and never married) were found to be potential factors that increased the risk of Paget's disease[31-33]. According to the previous study, for PD alone and patients with intraductal ductal carcinoma and breast cancer, breast conserving surgery combined with radiotherapy had a 5-year local recurrence rate of 5\% [34], which hintingthat surgery combined with radiotherapy could effectively affect the prognosis of Paget's disease. Our analysis was consistent with these previous reports.

Based on the above-mentioned risk factors, and considering the limitations of the traditional AJCC staging system, it may not be possible to predict the overall survival of Paget's disease better. Therefore, we constructed a nomogram by combining seven independent prognostic factors to predict the overall survival of Paget's disease. Our model found that the AJCC stage and age had a significant effect on the total score prediction. The $\mathrm{C}$-index and calibration curve were satisfactory when verified, indicating that the model is reproducible and reliable. In our nomogram, age $>51$ years and AJCC stage $>$ II grades scored higher, which indicated that patients with higher AJCC stage had worse prognosis as age increases after age 51. By comparing it with the traditional AJCC stage, we evaluated the value of this novel nomogram in predicting OS. Compared with the AJCC stage, our nomogram had better resolvability and accuracy in predicting 3-year and 5-year OS. In addition, using DCA, it had been fully demonstrated that the established nomogram could predict survival better than the AJCC staging system. Similarly, some studies had used DCA to validate benefits and models (clinical applications of predictive power [35, 36]). This was the first study to compare the newly established model with the traditional AJCC staging model and proved its better predictive ability for PD patients. We believed that our model would directly help clinicians to quantify the risk of cancer-specific deaths and to design more appropriate treatment strategies for PD patients.

\section{Limitation}

Our research also had some limitations that should be acknowledged. First, this was a large sample retrospective study based on the SEER database, which may have some inherent biases. Second, there were few potentially important parameters and specific information related to prognosis in the SEER database, such as family history of breast cancer, surgical margin status, vascular infiltration, radiotherapy and chemotherapy types. Third, we excluded patients who lacked data on collected variables, which could lead to selection bias. Fourth, because there were fewer cases of male Paget's disease in our screening, we did not include them in order to avoid confusion caused by imbalanced gender ratios, but it may also bring limitations. Fifth, cases of Paget disease complicated with invasive ductal carcinoma (PD-IDC) and Paget disease complicated with intraductal carcinoma (PD-DCIS) were not included in the cohort, so the reliability of the results may be reduced. Sixth, in order to avoid analysis bias due to missing cases, we chose to interval the cases from 1988 to 2015 , but the complication cases 
in the SEER database began to distinguish HER2 subtypes after 2010, so in our study some of the potential cases of HER2 proved to be unknown, which is one of the limitations of this study and has been observed in other literature. But, in the end, our nomogram was verified internally by Bootstrap resampling analysis.

\section{Conclusions}

In summary, to predict the prognosis of PD patients more accurately, we constructed and validated a nomogram that predicted 3-year and 5-year OS based on a large population-based cohort. The proposed nomogram considered seven independent risk factors, namely age, race, AJCC staging, marital status, chemotherapy, radiotherapy and surgery. By comparing it with the AJCC staging system, we confirmed the discriminative power and clinical utility of this nomogram.

\section{Declarations}

\section{Acknowledgements}

The authors would like to thank the SEER databases for the availability of the data and all the participants for their patience and cooperation

\section{Authors' contributions}

Conception and design: Kun Fang, Wei Li

Collection and assembly of data: Wei Li, Lin Long, Kang-yun Tang,

Data analysis and interpretation: Kun Fang, Wei Li, Guo Huang

Manuscript writing: Kun Fang, Wei Li, Li-zhi Su,Dan Li

Paper revision: Kun Fang, You-quan Wang, Hong Cao,Tian Zhu

Final approval of manuscript: All authors.

\section{Funding}

This study was funded by the Key R \& D projects in Ningxia, China (2018BEG03081)and Clinical Research Center for Prevention and Treatment of Breast \& Thyroid Disease in Hunan,China『2018SK4001区.

\section{Availability of data and materials}

The datasets generated and/or analyzed during the current study are available from the corresponding author on reasonable request. 
Not applicable.

\section{Consent for publication}

Not applicable.

\section{Competing interests}

The authors declare that they have no conflict of interest.

\section{References}

1. Siegel RL, Miller KD, Jemal A. Cancer statistics, 2016. CA Cancer J Clin. 2016;66:7-30.

2. Eckhardt BL, Francis PA, Parker BS, Anderson RL. Strategies for the discovery and development of therapies for metastatic breast cancer. Nat Rev Drug Discov. 2012;11:479-497.

3. Kennecke H, Yerushalmi R, Woods R, Cheang MC, Voduc D, Speers CH, Nielsen TO, Gelmon K. Metastatic behavior of breast cancer subtypes. J Clin Oncol. 2010;28:3271-3277.

4. Redig AJ, McAllister SS. Breast cancer as a systemic disease: a view of metastasis. J Intern Med. 2013;274:113-126.

5. Zakaria S, Pantvaidya G, Ghosh K, Degnim AC. Paget's disease of the breast: accuracy of preoperative assessment. Breast Cancer Res Treat. 2007;102(2):137-142.

6. Dalberg K, Hellborg H, Wärnberg F. Paget's disease of the nipple in a population based cohort. Breast Cancer Res Treat. 2008;111(2): 313-319.

7. Trebska-Mcgowan K, Terracina KP, Takabe K. Update on the surgical management of Paget's disease. Gland Surg. 2013;2(3).

8. Wong SM, Freedman RA, Sagara Y, et al. The effect of Paget disease on axillary lymph node metastases and survival in invasive ductal carcinoma. Cancer. 2015;121(24):4333-4340.

9. Helme S, Harvey K, Agrawal A. Breast-conserving surgery in patients with Paget's disease. Br J Surg. 2015;102(10):1167-1174.

10. Lim HS, Jeong SJ, Lee JS, et al. Paget disease of the breast: mammographic, US, and MR imaging findings with pathologic correlation. Radiographics. 2011;31(7):1973-1987.

11. Lopes Filho LL, Lopes IM, Lopes LR, Enokihara MM, Michalany AO, Matsunaga N. Mammary and extramammary Paget's disease. Anais brasileiros de dermatologia. Mar. 2015;90(2):225-231.

12. Muttarak M, Siriya B, Kongmebhol P, Chaiwun B, Sukhamwang N. Paget's disease of the breast: clinical, imaging and pathologic findings: a review of 16 patients. Biomed Imaging Interv $\mathrm{J}$. 2011;7(2):e16.

13. Sandoval-Leon AC, Drews-Elger K, Gomez-Fernandez CR, Yepes MM, Lippman ME. Paget's disease of the nipple. Breast Cancer Res Treat. 2013;141(1):1-12. 
14. 1 lasonos A, Schrag D, Raj GV, Panageas KS. How to build and interpret a nomogram for cancer prognosis. J Clin Oncol. (2008) 26:1364-70. doi: 10.1200/JC0.2007.12.9791.

15. Xiong Z, Deng G, Huang X, Li X, Xie X, Wang J, et al. Score for the survival probability in metastasis breast cancer: a nomogram-based risk assessment model. Cancer Res Treat. (2018) 50:1260-9. doi: 10.4143/crt.2017.443.

16. Lee SB, Sohn G, Kim J, Chung IY, Lee JW, Kim HJ, et al. A retrospective prognostic evaluation analysis using the 8th edition of the American Joint Committee on Cancer staging system for breast cancer. Breast Cancer Res Treat. (2018) 169:257-66. doi: 10.1007/s10549-018-4682-5.

17. Plichta JK, Ren Y, Thomas SM, Greenup RA, Fayanju OM, Rosenberger LH, et al. Implications for breast cancer restaging based on the 8th edition AJCC staging manual. Ann Surg. (2018). doi: 10.1097/SLA.0000000000003071.

18. Kramer AA, Zimmerman JE. Assessing the calibration of mortality benchmarks in critical care: the Hosmer-Lemeshow test revisited. Crit Care Med. 2007;35(9):2052-2056.

doi:10.1097/01.CCM.0000275267.64078.B0 19.Leibou L, Herman O, Frand J, et al. Paget's disease of the male breast with underlying ductal carcinoma in situ[J]. Isr Med Assoc J, 2015,17(1):64-65.

19. Adams S J, Kanthan R. Paget's disease of the male breast in the 21 st century: A systematic review[J]. Breast, 2016,29:14-23.

20. Jia Linjiao, Zhai Baoping, Li Wentao, et al. A case of para-breast pieger's disease [J]. Chinese Journal of Breast Disease (Electronic Edition),2013, 7 (02): 146-147.

21. Helme S, Harvey K, Agrawal A. Breast-conserving surgery in patients with Paget's disease[J]. British Journal of Surgery, 2015,102(10):1167-1174.

22. Paone J F, Baker R R. Pathogenesis and treatment of Paget's disease of the breast[J]. Cancer, 1981,48(3):825-829.

23. Wan Miaojian, Ma Han, Gong Zijian, et al. Mohs micrograph surgery of surrounding tissues for the treatment of extra-mammary Paget disease [J]. Chinese Journal of Dermatology, 2016,49 (4): 278280.

24. Plichta JK, Ren Y, Thomas SM, Greenup RA, Fayanju OM, Rosenberger LH, et al. Implications for breast cancer restaging based on the 8th edition AJCC staging manual. Ann Surg. (2018).

25. Liu RZ, Zhao ZR, Ng CS. Statistical modelling for thoracic surgery using a nomogram based on logistic regression. J Thorac Dis. 2016;8(8):E731-E736. doi:10.21037/jtd.2016.07.91

26. Balachandran VP, Gonen M, Smith JJ, DeMatteo RP. Nomograms in oncology: more than meets the eye. Lancet Oncol. 2015;16:e173-80.

27. Iasonos A, Schrag D, Raj GV, Panageas KS. How to build and interpret a nomogram for cancer prognosis. J Clin Oncol. 2008;26:1364-70.

28. Shariat SF, Karakiewicz PI, Suardi N, Kattan MW. Comparison of nomograms with other methods for predicting outcomes in prostate cancer: a critical analysis of the literature. Clin Cancer Res. 2008;14:4400-7. 
29. Yim JH, Wick MR, Philpott GW, Norton JA, Doherty GM. Underlying pathology in mammary Paget's disease. Ann Surg Oncol. 1997;4(4):287-292. doi:10.1007/bf02303576

30. Martin VG, Pellettiere EV, Gress D, Miller AW. Paget's disease in an adolescent arising in a supernumerary nipple. J Cutan Pathol. 1994;21:283-6

31. Bennett T. 1992. Marital status and infant health outcomes. Soc. Sci. Med. 35:1179-1187.

32. Johnson N. J., Backlund E., Sorlie P. D., and Loveless C. A.. 2000. Marital status and mortality: the national longitudinal mortality study. Ann. Epidemiol. 10:224-238.

33. Bijker N, Rutgers E J, Duchateau L, et al. Breast-conserving therapy for Paget disease of the nipple: a prospective European Organization for Research and Treatment of Cancer study of 61 patients[J]. Cancer, 2001,91(3):472-477.

34. Liang W, Yang P, Huang R, et al. A Combined Nomogram Model to Preoperatively Predict Histologic Grade in Pancreatic Neuroendocrine Tumors. Clin Cancer Res. 2019;25(2):584-594. doi:10.1158/1078-0432.CCR-18-1305

35. Van Calster B, Wynants L, Verbeek JFM, et al. Reporting and Interpreting Decision Curve Analysis: A Guide for Investigators. Eur Urol. 2018;74(6):796-804. doi:10.1016/j.eururo.2018.08.038

\section{Tables}

Due to technical limitations, Tables $1 \& 2$ are only available for download from the Supplementary Files section.

\section{Figures}


Points

$\begin{array}{lllllllllll}0 & 10 & 20 & 30 & 40 & 50 & 60 & 70 & 80 & 90 & 100\end{array}$

Age

Race

AJCC stage

Surgery

Radiotherapy

Chemotherapy

Marital status
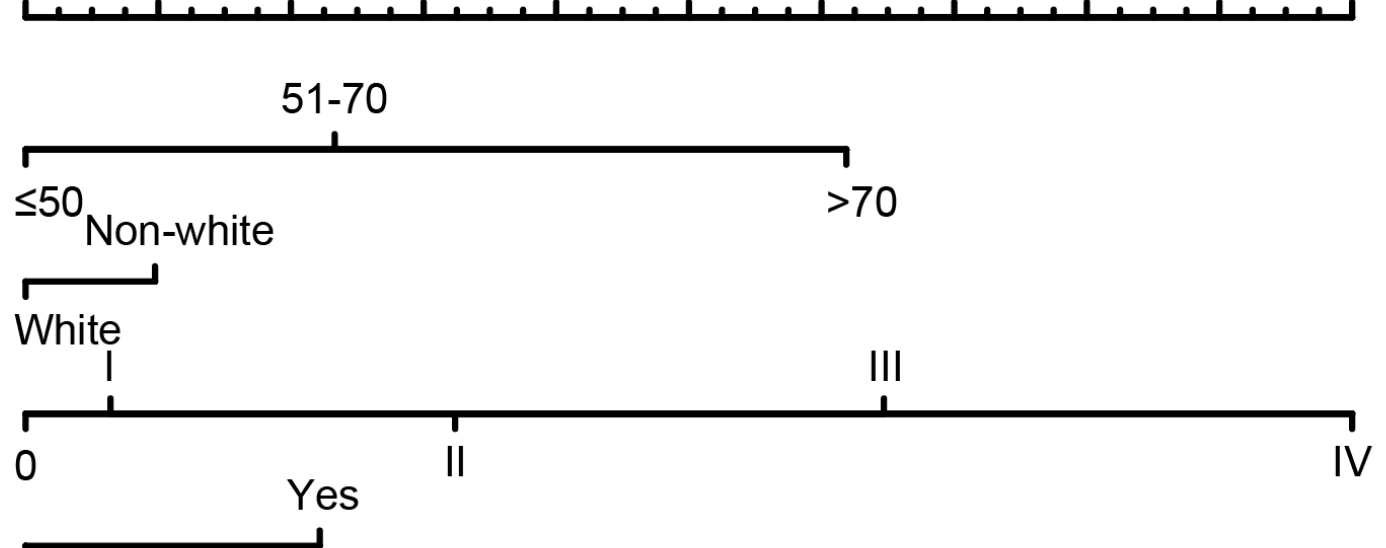

Total Points

1-year survival
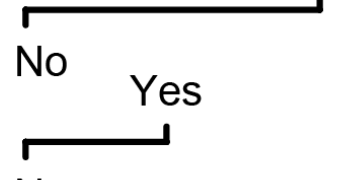

No

Yes

3-year survival

No

Unknown

$\longmapsto$

Single Married

5-year survival

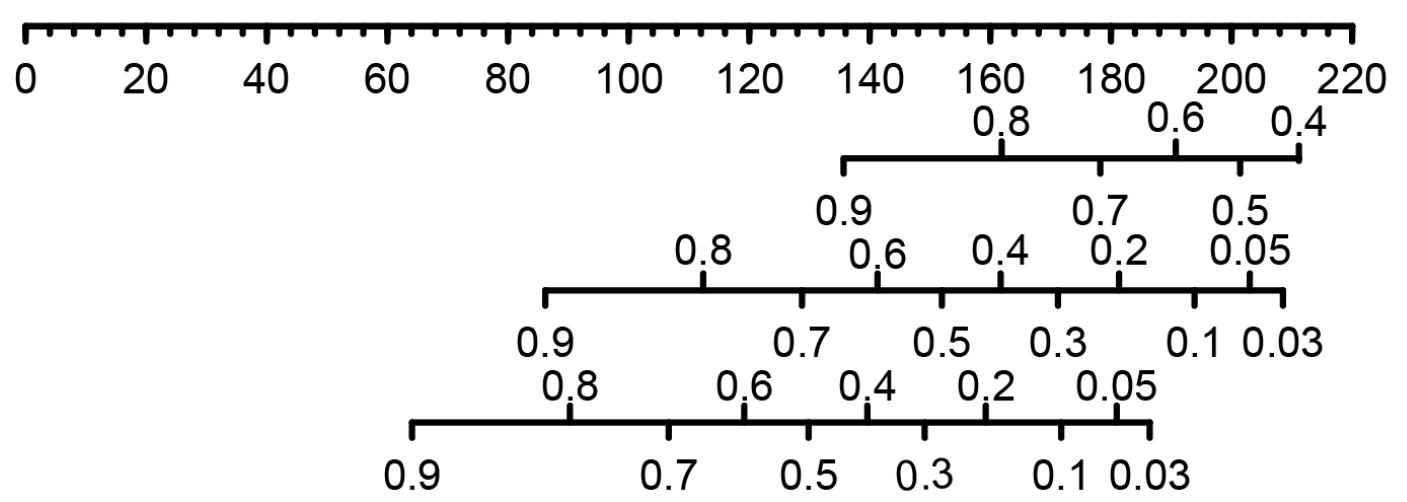

Figure 1

Nomogram predicts 1-, 3-, and 5-year PD overall survival rates for PD patients with six available factors, including race, American Cancer Council (AJCC) staging, surgery, radiation therapy, chemotherapy, and marital status. 


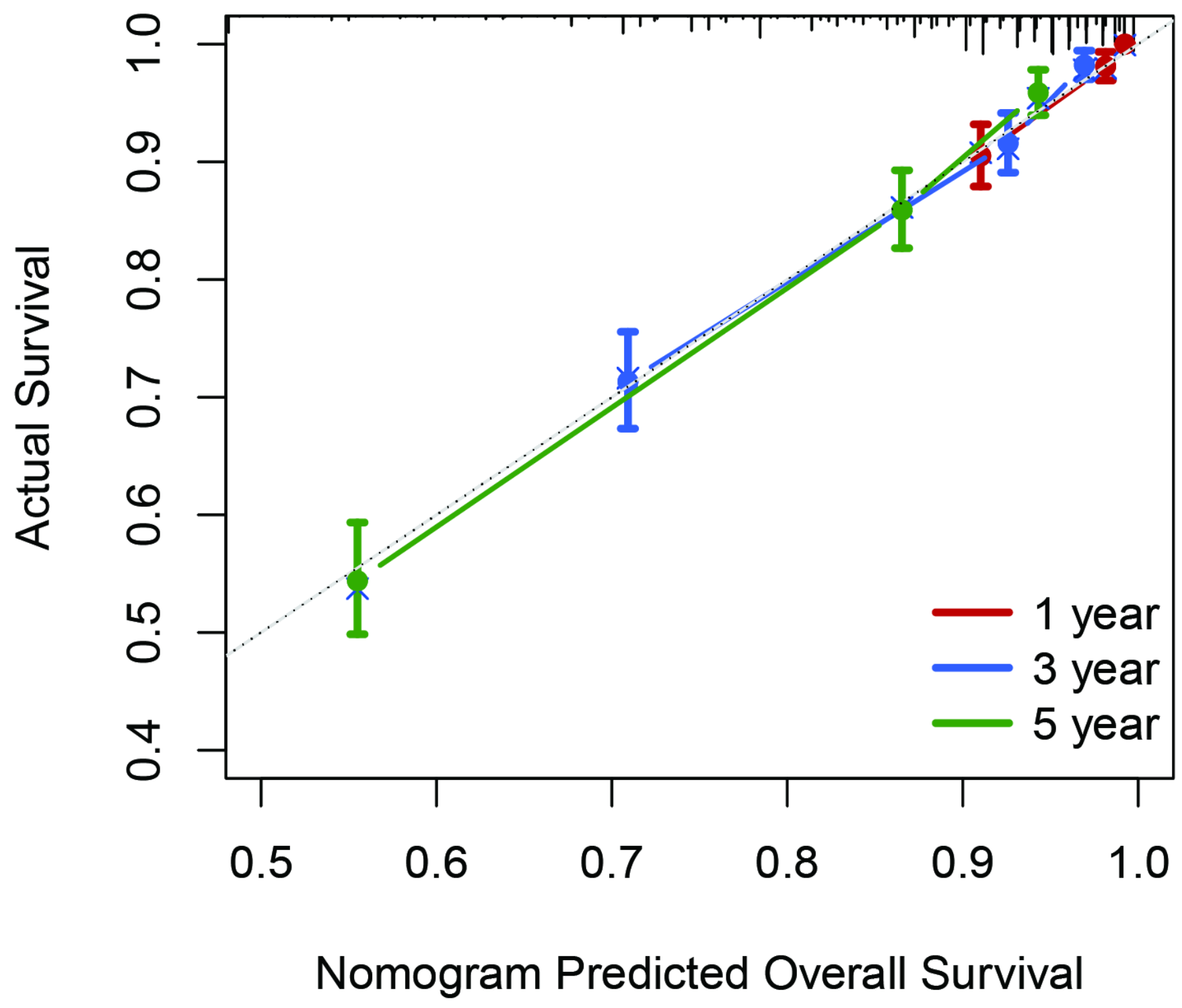

Figure 2

Calibration curves for predicting 1-, 3- and 5-year OS in patients with PD. The nomogram-predicted probability of survival is plotted on the $\mathrm{X}$-axis, and the actual survival rate is plotted on the $\mathrm{Y}$-axis. Vertical bars indicate $95 \%$ Cls measured by Kaplan-Meier analysis. 

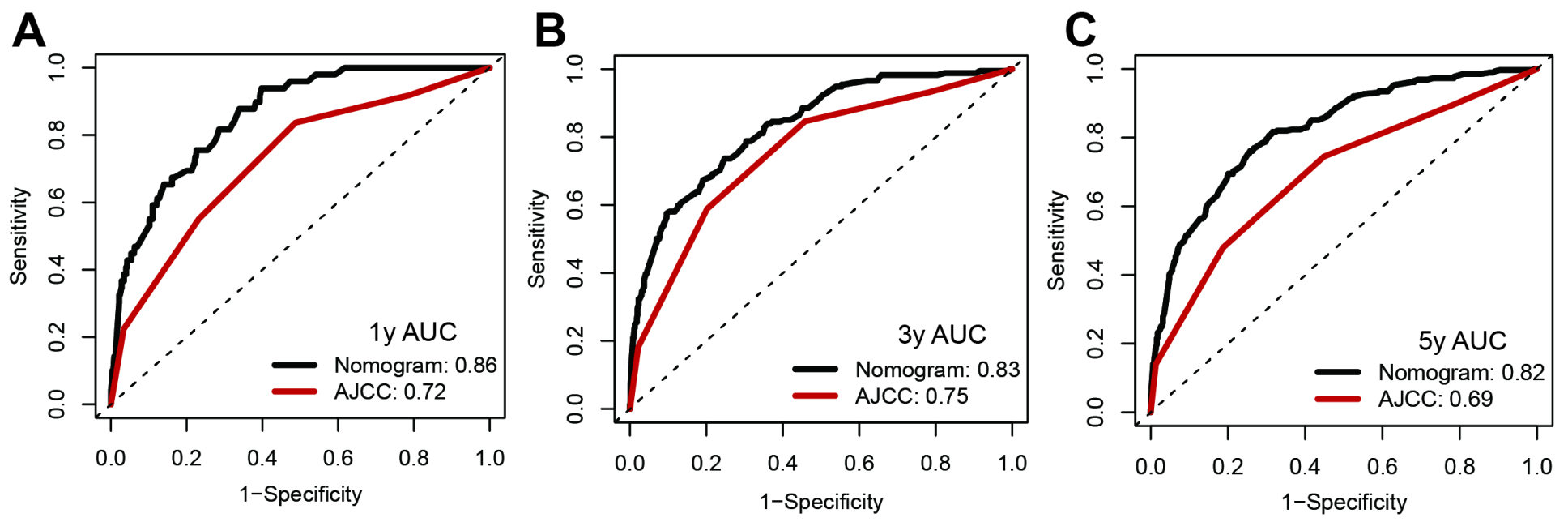

Figure 3

ROCs curve demonstrates sensitivity and specificity of nomogram VS AJCC. The AUC values used to compare the sensitivity and specificity between nomogram and AJCC predicted 1-, 3-, and 5-year OS of PD patient

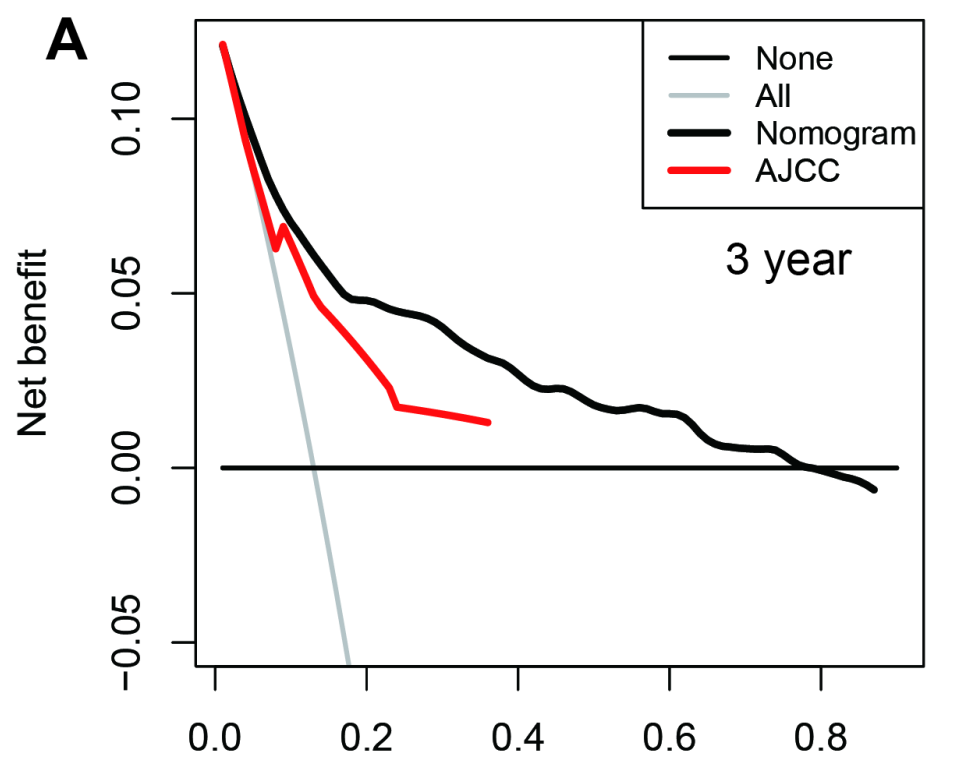

Threshold probability

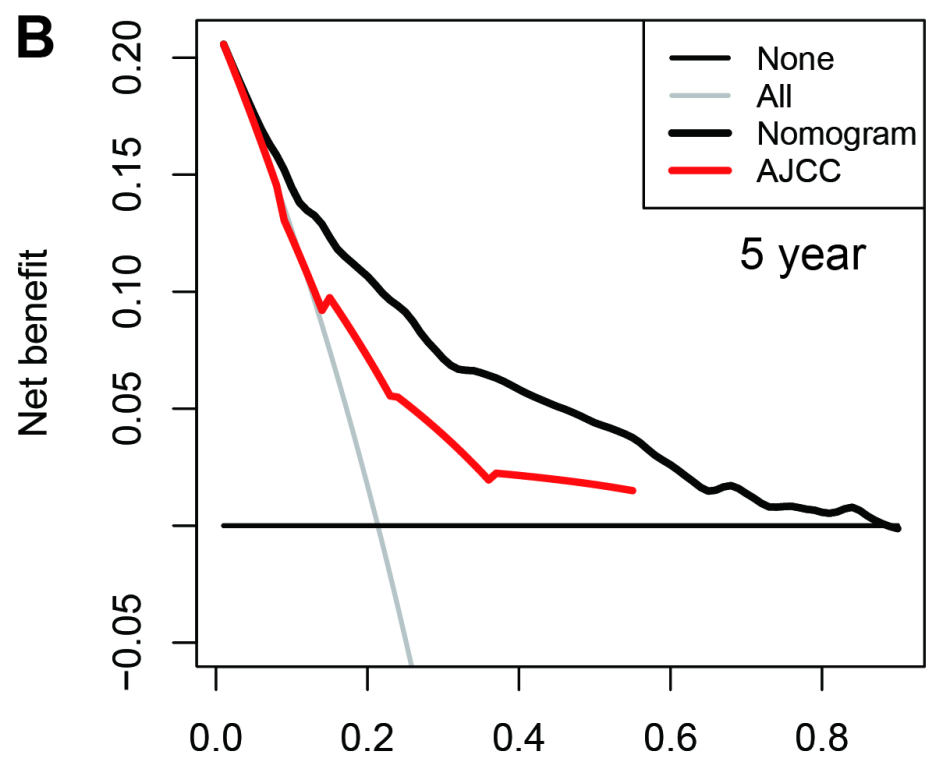

Threshold probability

Figure 4

Decision curve analysis for the Nomogram and AJCC stage in prediction of prognosis of male patients at 3-year (A) and 5-year (B) point in the validation cohorts.

\section{Supplementary Files}

This is a list of supplementary files associated with this preprint. Click to download. 
- Table2.docx

- Table1.docx 